\title{
Simulation der Rauchgasströmung in einem Kessel zur Müllverbrennung
}

Udo Hellwig, Hartwig Nikolaus

\section{Allgemeines}

Im Bereich der Industriekesseltechnik besteht ein großes Optimierungspotential hinsichtlich der gas- und wasserseitigen Strömungsführung, um die Prozesse effizienter gestalten zu können. Dampferzeuger wurden lange Zeit auf sehr traditionelle Art und Weise ausgelegt. Rechentechnische Modelle zur Beschreibung komplexer Vorgänge lagen kaum vor. Die vorhandenen ermöglichten nur eine stark vereinfachte Darstellung der Wirklichkeit.

Die numerische Berechnung von physikalischen Phänomenen der Strömungsmechanik hat sich zunehmend von einem reinem Hilfsmittel der Wissenschaft zu einem Ingenieurwerkzeug entwickelt. Neben dem Experiment steht damit die CFD- (Computational Fluid Dynamics) Simulation als weiteres Problemlösungswerkzeug zur Verfügung. CFD-Analysen ermöglichen Einblick und damit ein tieferes Verständnis von Strömungs-, Wärme- und Stoffuibertragungsvorgängen und weiteren physikalischen Prozessen.

Besonders in der Kesseltechnik liegt ein ausgedehntes Anwendungsgebiet für diese Berechnungsmethoden vor, da

- eine meßtechnische Erfassung relevanter Parameter oft nur eingeschränkt möglich ist und
- hohe meßtechnische Anforderungen durch Hochtemperaturbereiche, korrosive Medien (Gasseite) und Zweiphasenströmungen (Wasser/Dampfseite) gestellt werden.

CFD wird in zunehmendem Maße ein integraler Bestandteil bei der Auslegungs- sowie bei der Optimierungsarbeit bestehender Wärmeübertragungs- und Verbrennungssysteme.

\section{Ziele der Simulation}

Durch eine CFD-Simulation sollen die Ergebnisse üblicher wärmetechnischer Berechnungen (ERK-Temperaturmethode) auf ein dreidimensionales strömungstechnisches Modell übertragen werden. Die uiblichen eindimensionalen Berechnungen liefern in der Regel belastbare Ergebnisse mit dem Nachteil, daß nur eine „überschlägige" Betrachtung möglich ist und lokale Gegebenheiten nicht aufgelöst werden können.

Eine CFD-Berechnung ermöglicht neben der quantitativen Auswertung relevanter strömungs- und verbrennungstechnischer Parameter an jeder Stelle des Berechnungsgebietes eine anschauliche Visualisierung der Strömungsgrößen in Form von farbigen Grafiken und Animationen.

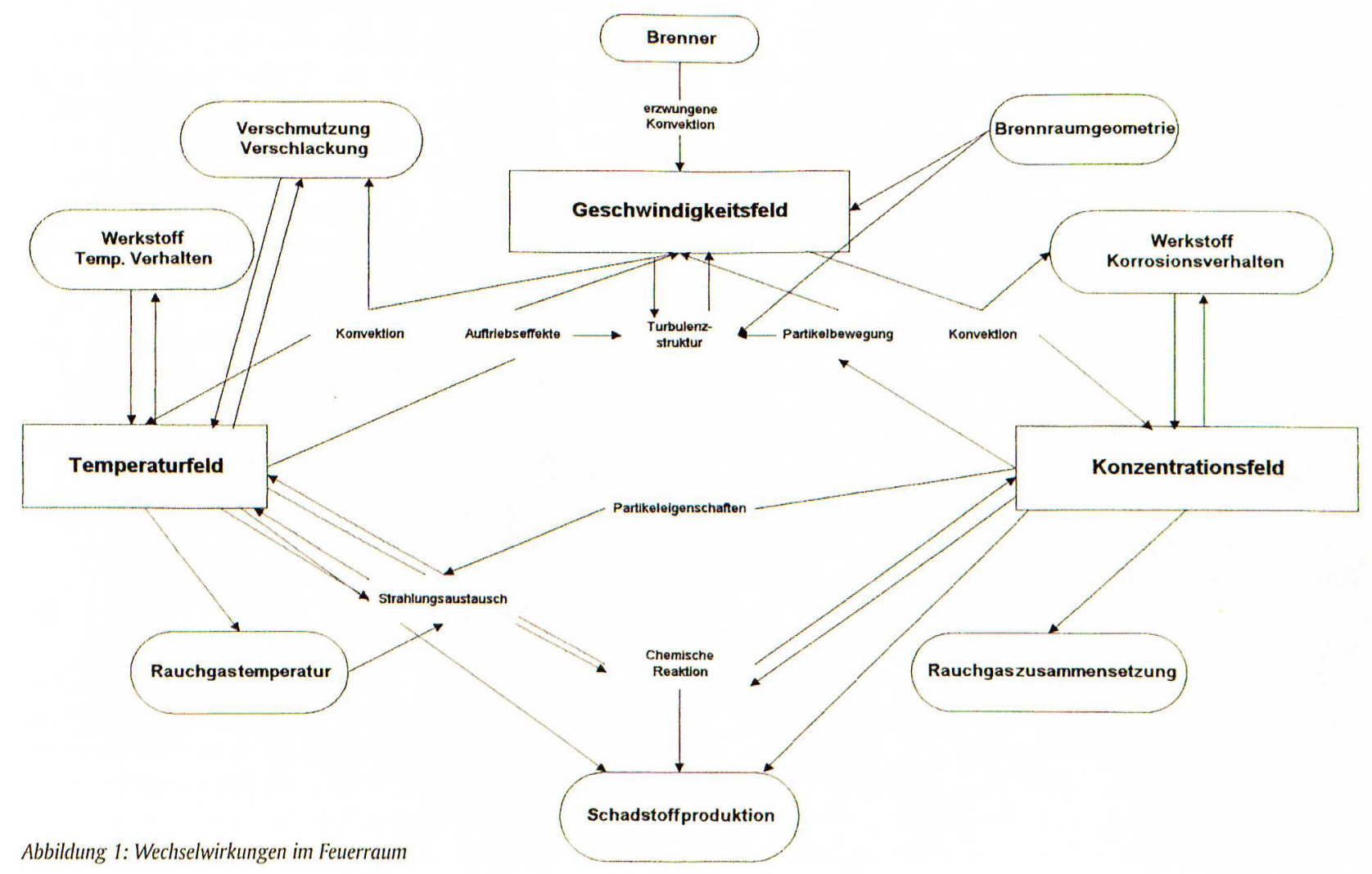


Die strömungstechnische Bewertung des Geschwindigkeits- und Temperaturfeldes läßt Rückschliisse auf relevante Betriebsgrößen zu, wie:

- stoffliche Umsatzraten,

- Partikelausbrand bzw. -absetzverhalten,

- Erosion- und Korrosionsmechanismen,

- Wärmeübertragungsverhalten.

\section{Randbedingungen des CFD-Modells}

\subsection{Wechselwirkungen bei der Mülllverbrennung}

Die Vorgänge im Feuerraum eines Dampferzeugers sind sehr komplex. Abbildung1 nach Görner [1] gibt einen Überblick der Wechselwirkungen im gesamten technischen System.

Es beeinflussen sich dabei die Geschwindigkeits-, Temperatur- und Konzentrationsverteilungen der einzelnen Spezies gegenseitig.

Das dreidimensionale Geschwindigkeitsfeld ist durch die hohen Impulse, die durch die Verbrennung und die Sekundärlufteinduisung eingebracht werden, turbulent. Die Art der Turbulenz unterscheidet sich aber signifikant von der einer Strömung in langen Rohren.

- Das Temperaturfeld wird im Wesentlichen durch die Wärmeentbindung bei der Verbrennung und das Wärmeübertragungsverhalten des Feuerraums beeinflußt.

- Die Konzentrationsfelder sind direkt von den obigen Feldern abhängig.

Das Feuerraummodell läßt sich demnach in die Submodelle Strömungsmodell, Wärmeübertragermodell und Reaktionsmodell gliedern. Wie eingangs erwähnt, beschränken sich die hier vorgestellten Arbeiten auf die Strömungs- und Wärmeübertragungsseite.

Bei der Erfassung der Strömungssituation durch die CFD-Simulation muß berüicksichtigt werden, daß die Rostfeuerung aufgrund des stark inhomogenen Brennstoffs „Restmuill“ ein stark dynamisches Verhalten aufweist. Die Regelungstechnik reagiert auf veränderte Bedingungen im Feuerraum durch Veränderungen der Walzendrehgeschwindigkeit, Verbrennungsluftmenge, Aufteilung der Verbrennungsluft in Primär- bzw. Sekundärluftanteile etc.

Ein wichtiger Parameter ist in diesem Zusammenhang die Feuerlage, d.h. der Schwerpunkt der maximalen Wärmeentbindung. Sie repräsentiert die Wärmeentbindung auf dem Rost. Veränderte Betriebspunkte führen zu unterschiedlichen Strömungs- und Reaktionssituationen im Feuerbereich und dadurch zu differierenden Wärmeentbindungen an den Heizflächen.

Eigene CFD-Untersuchungen haben allerdings gezeigt, daß die Feuerlage in der Regel keinen erheblichen Einfluß auf die Strömung nach der letzten Sekundärlufteinduisung im Feuerraum hat [2].

Die Verweilzeit der Gase im Feuerraum liegt in Abfallverbrennungsanlagen im Bereich von mehreren Sekunden, während die Verweilzeit des Mülls auf dem Rost bis zu mehrere Stunden beträgt. Damit kann in Bezug auf die Müilloberfläche von einem gleichbleibenden Einfluß auf die Gasströmung ausgegangen werden. Durch die vorliegende CFD-Simulation wird ein stationärer Fall beschrieben, d.h. die Strömungsvorgänge werden lokal als zeitlich unveränderlich angesehen. Instationäre Simulationen sind grundsätzlich möglich, sie erfordern aber einen erheblichen zeitlichen und auch darstellerischen Aufwand.

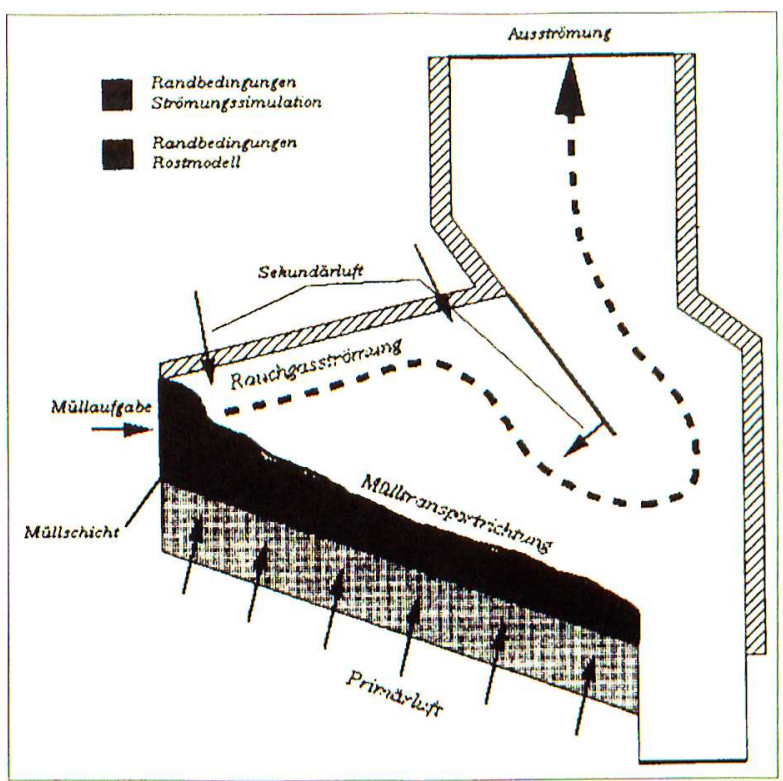

Abbildung 2: Prinzipdarstellung der Randbedingungen für die Feuerraumsimulation (Gleichstromfeuerung)

\subsection{Geometrie und Berechnungsgitter}

CFD-Software verwendet bis auf wenige Ausnahmen das Modell der Finiten Differenzen, wobei das Berechnungsgebiet in sogenannte Kontrollvolumina aufgeteilt wird. Innerhalb der Kontrollvolumina werden die Erhaltungsgleichungen für Masse, Impuls und Energie gelöst. Die turbulenten Fluktuationen innerhalb der Strömung werden im technischen Maßstab in der Regel durch statistische Methoden (Turbulenzmodelle) beschrieben. Die Betrachtungen in der vorgestellten Simulation beschränken sich auf die Zustände auf der Gasseite der Müllverbrennungsanlage (Abbildung 3).

Das Berechnungsgebiet wurde im ersten Schritt durch 170.000 Zellen in Hexaederform diskretisiert. Im Rahmen der Simulationsrechnung wurde das Gitter im Bereich der Sekundärlufteindüsung auf Basis der vorherrschenden Geschwindigkeitsgradienten adaptiert, so daß die abschließende Gitterzellenzahl bei 200.000 Zellen lag (Abbildung 4).

\subsection{Vorgehensweise}

Als Basis für die CFD-Simulation wurden die Daten einer eindimensionalen wärmetechnischen Berechnung verwendet, wie sie im Kesselbau zur Heizflächenauslegung uiblich ist. Dabei werden sowohl auf der Rauchgasseite als auch auf der Wasser-Dampfseite alle Heizflächen des Kessels bilanziert. Die Größen der 


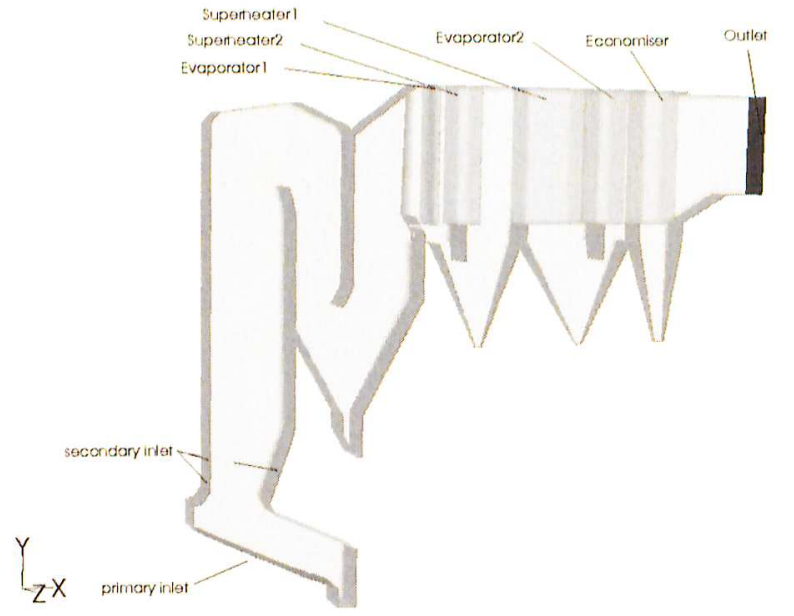

Abbildung 3: Geometrie des CFD-Modells

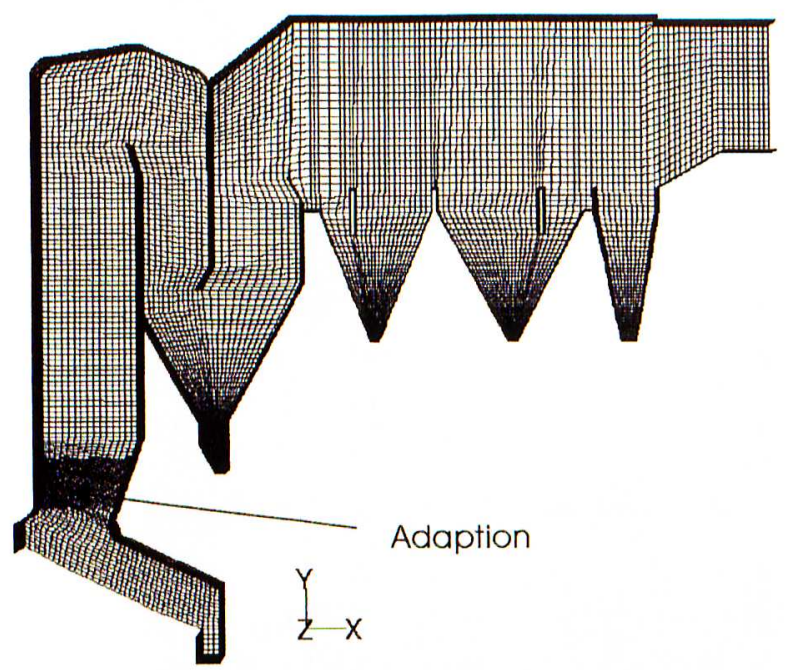

Abbildung 4: Berechnungsgitter

Wärmeübertragung werden nach einem Verfahren gerechnet, das sich über Jahrzehnte bewährt hat. Der gesamte Kessel wird auch bilanziert und die erforderliche Brennstoffmenge aus dem Wirkungsgrad ermittelt. Für die Verluste werden ïbliche Werte für den Brennstoff und die Kesselgröße eingesetzt.

Die Geometriedaten zur Erstellung der 3-D-Konstruktion wurden mit Hilfe vorliegender Zeichungssätze zusammengestellt. Fehlende Maße wurden durch Maßentnahmen ergänzt.

\subsection{Projektbezogene Annahmen}

Zur Vereinfachung der Randbedingungen wurden für das CFD-Modell folgende Annahmen getroffen:

- Die Berechnung erfolgt aufgrund der vorhandenen Symmetrie im Halbschnitt.

- Die Berechnung erfolgt ohne chemische Reaktion (Vergasung/Verbrennung).

- Die Berechnung erfolgt mit drei Sekundärluftreihen mit sieben, acht bzw. neun Düsen, die unterschiedliche Anstellwinkel in der vertikalen Ebene aufweisen.

- Die Heizflächen im Kessel werden als poröse Medien definiert. Den porösen Medien werden rechnerisch ermittelte Widerstandsbeiwerte in allen drei Raumrichtungen zugewiesen, die einen Impulsverlust initiieren. In diesem Bereich wird eine konstante Wärmesenke vorgegeben.

- Die Verbrennungsluft wird in einen Primärluftanteil von ca. 2/3 und einen Sekundärluftanteil von ca. 1/3 aufgeteilt.

- Primär- und Sekundärluft werden mit unterschiedlichen Temperaturniveaus in das System eingebracht. Durch die Sekundärluftduisen strömt Umgebungsluft ein. Die Zusammensetzung der Primärluft $\left(\mathrm{O}_{2}, \mathrm{CO}_{2}\right.$, $\mathrm{H}_{2} \mathrm{O}$ und $\mathrm{N}_{2}$ ) wird so gewählt, daß sich nach dem Feuerraum die in der eindimensionalen Berechnung ermittelte Rauchgaszusammensetzung einstellt.

- Um die Temperaturverhältnisse auf dem Rost abbilden zu können, wurde dieser in vier Zonen unterteilt (Tabelle 1). Es wurde eine realistische Temperaturverteilung auf dem Rost angenommen, die eine Wärmeentbindung durch den Verbrennungsprozess widerspiegelt. Die Vermischung der eintretenden Primärluft ergibt ein Rauchgas mit dem Temperaturniveau, welches der adiabaten Verbrennungstemperatur entspricht. Diese liegt für den Voll- und Teillastfall etwa in der gleichen Größenordnung.

\begin{tabular}{|c|c|c|c|}
\hline & & Vollast & Telllast \\
\hline Nr. & $\begin{array}{l}\text { Prozentualer Anteil } \\
\text { an der Primärluft }\end{array}$ & $\begin{array}{c}\text { Temperatur } \\
{ }^{\circ} \mathrm{C}\end{array}$ & $\begin{array}{c}\text { Temperatur } \\
{ }^{\circ} \mathrm{C}\end{array}$ \\
\hline Zone 1 & $26 \%$ & 527 & 503 \\
\hline Zone 2 & $33 \%$ & 2283 & 2177 \\
\hline Zone 3 & $29 \%$ & 2634 & 2512 \\
\hline Zone 4 & $12 \%$ & 702 & 700 \\
\hline
\end{tabular}

Tabelle 1: Temperaturniveaus der Primärluftzonen

\begin{tabular}{|c|c|c|c|c|c|c|}
\hline & \multicolumn{3}{|c|}{ Vollast } & \multicolumn{3}{|c|}{ Telllast } \\
\hline & Primarluft & Sekundärluft & Rauchgas & Primárluft & Sekundäıluft & Rauchgas \\
\hline & \multicolumn{3}{|c|}{ Massenanteile in $\%$} & \multicolumn{3}{|c|}{ Massenanteile in \% } \\
\hline $\mathrm{CO}_{2}$ & 14,68 & - & 9.04 & & - & 8.36 \\
\hline $\mathrm{H}_{2} \mathrm{O}$ & 25,76 & - & 15,86 & & - & 13,33 \\
\hline $\mathrm{N}_{2}$ & 59,54 & 79 & 67,02 & & 79 & 68.98 \\
\hline \multirow[t]{3}{*}{$\mathrm{O}_{2}$} & 0,01 & 21 & 8.08 & & 21 & 9.33 \\
\hline & \multicolumn{3}{|c|}{ volumenstrome in $\mathrm{m}^{3} / \mathrm{h}$} & \multicolumn{3}{|c|}{ Volumenstrome in $\mathrm{m}^{3} / \mathrm{h}$} \\
\hline & 18.126 & 11.316 & 29.442 & 10.598 & 6.616 & 17.214 \\
\hline
\end{tabular}

Tabelle 2: Aufteilung der Volumenströme (Halbschnitt)

\section{Strömungstechnische Bewertung}

Ein optimales Design des Kessels ermöglicht die Ausbildung eines gleichmäßigen vertikalen Geschwindigkeitsfeldes bei guter Durchmischung des Rauchgases durch die Sekundärlufteindüsungen. In diesem Fall werden Rezirkulationen, Partikelablagerungen und Rauchgassträhnen mit hoher Geschwindigkeit weitestgehend vermieden.

Aus der Bewertung des Strömungsfeldes läßt sich das Verhalten anderer Größen, die für den Betrieb der Kesselanlage relevant sind, ableiten. Hierzu zählen z.B. die reaktionstechnischen Vorgänge, der Transport von Staub sowie dessen Abscheidung und das Wärmeübertragungsverhalten.

Die Kesselanlage läßt sich aus strömungstechnischer Sicht in vier kritische Teilbereiche gliedern (Abbildung 5).

Diese vier Bereiche werden im Folgenden auf ihre strömungstechnische Wirksamkeit hin untersucht. Auf der Basis der strömungstechnischen Situation und der 


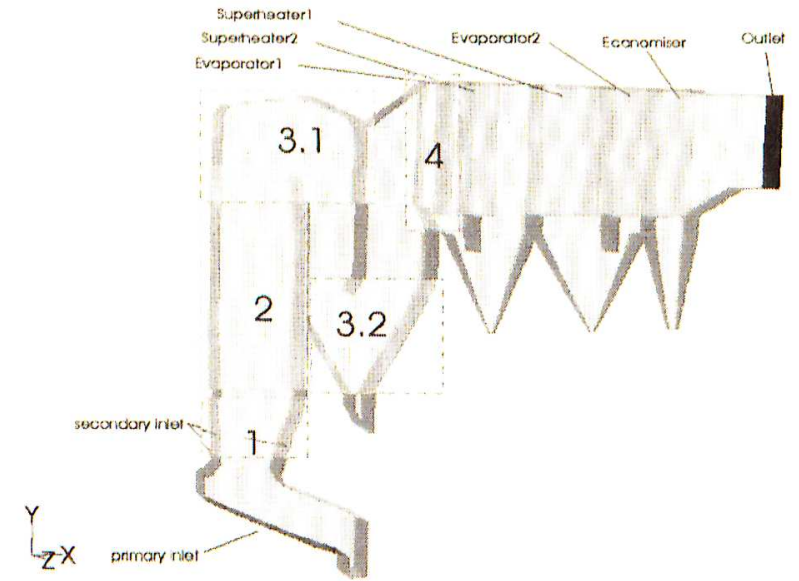

Abbildung 5: Unterteilung des Strömungsgebietes in Teilbereiche

Temperaturverteilungen werden Aspekte wie die reaktionstechnische Wirksamkeit und das Partikelabsetzverhalten diskutiert.

Die Visualisierung der relevanten Strömungsgrößen und deren Interpretation beschränken sich ausschließlich auf den Vollastfall. Der Vergleich mit dem Teillastfall hat die Annahme bestätigt, daß sich aufgrund der gleichen Relationen zwischen Primär- und Sekundärluft sowie der Temperaturverteilung auf dem Rost ein nahezu identisches strömungstechnisches Gesamtbild einstellt.

In Kap. 5 wird ein Vorschlag fuir die Modifikation der Aufteilung der Sekundärluft auf die einzelnen Düsenreihen vorgestellt.

\section{Sekundärlufteindüsung}
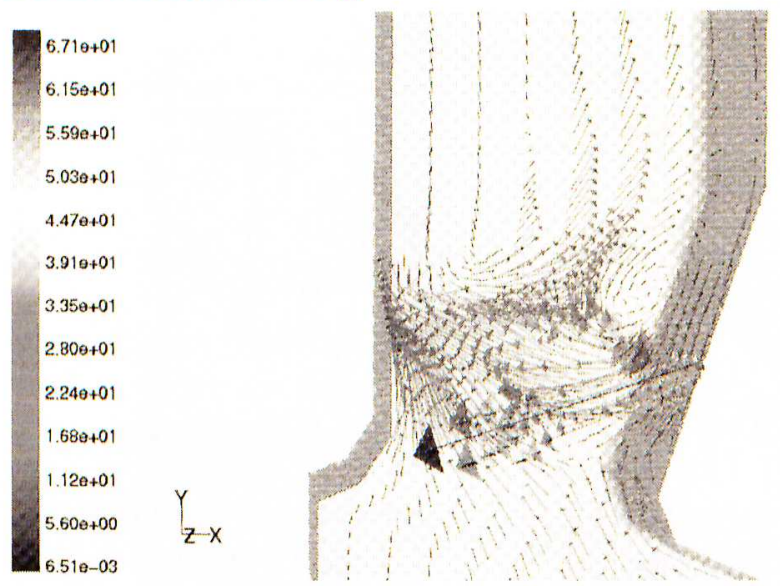

-Abbildung 6: Geschwindigkeitsvektoren in m/s, Symmetrie (gefärbt durch den Betrag der Geschwindigkeit)

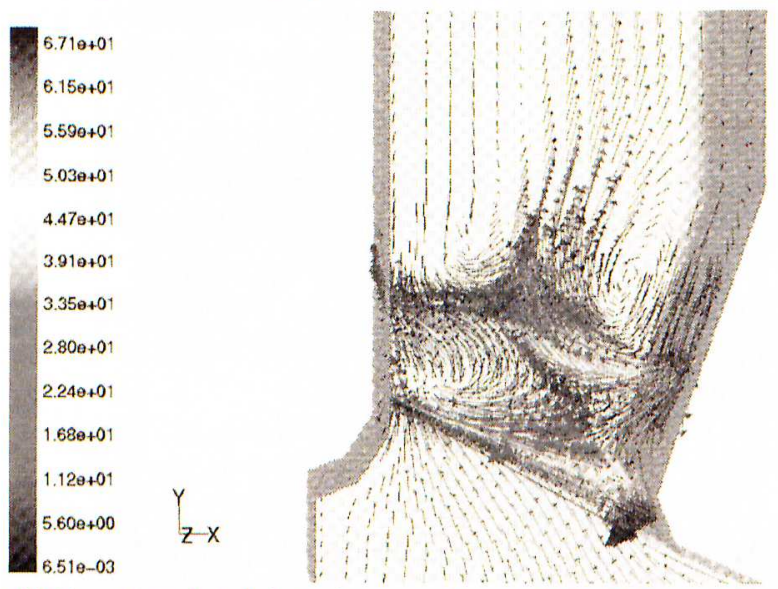

Abbildung 7: Geschwindigkeitsvektoren in $\mathrm{m} / \mathrm{s}, z=1.600$ (gefärbt durch den Betrag der Geschwindigkeit)

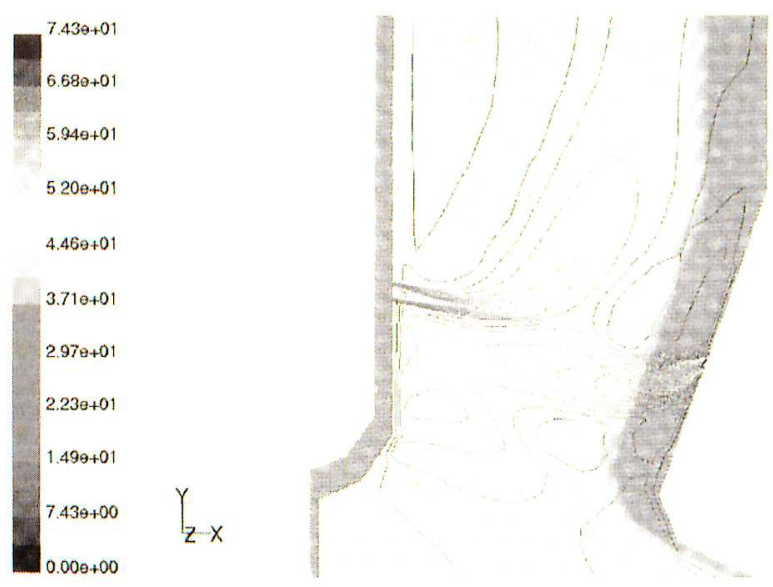

Abbildung 8: Geschwindigkeitskonturen in $\mathrm{m} / \mathrm{s}$, Symmetrie

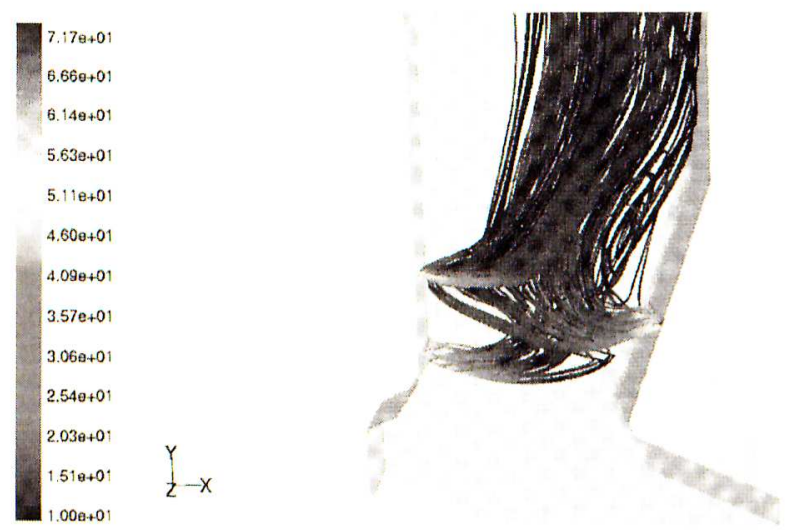

Abbildung 9: Stromlinien gefärbt durch die Geschwindigkeit in m/s

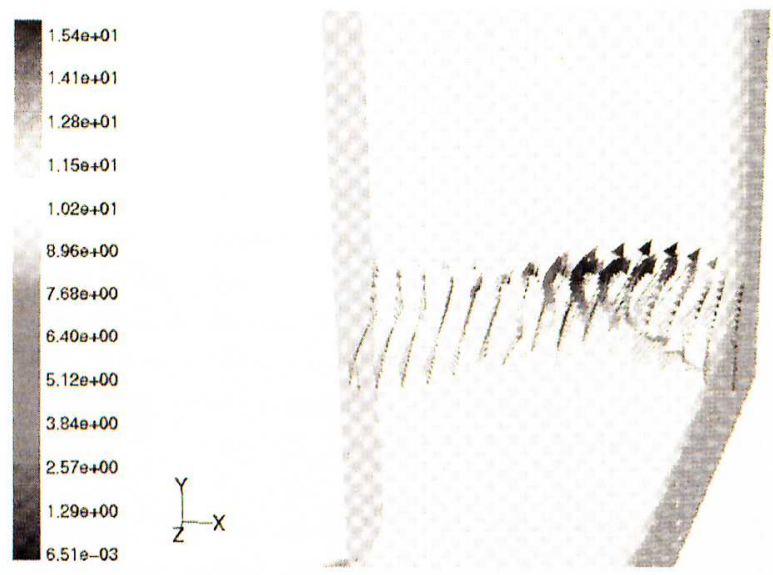

Abbildung 10: Geschwindigkeitsvektoren hinter der Sekundärlufteindüsung in m/s (gefärbt durch den Betrag der Geschwindigkeit)

\section{Geschwindigkeitsfeld}

Die Aufteilung der Verbrennungsluft in Primär- und Sekundärluft wurde aus strömungstechnischer Sicht in einem ausgewogenen Verhältnis auf der Grundlage von Impulsausgleichen gewählt. Der Gesamtimpuls der einströmenden Sekundärluft ist ausreichend, um eine gute Vermischung der Sekundärluft zu initiieren.

Abbildung 9 zeigt, daß die Eindringtiefe der Sekundärluftstrahlen ausreichend ist. Das bedeutet, der gesamte Strömungsquerschnitt der Hauptströmung wird durch die Sekundärlufteindüsung gestört.

Im vertikalen Schnitt nach der Sekundärlufteindüsung (Abbildung 10) ist zu erkennen, daß sich eine Einschniirung der Strömung einstellt. Diese Einschnürung kann durch eine modifizierte Luftstufung, d.h. die Aufteilung 
der Sekundärluft auf die einzelnen Düsenreihen verhindert werden. Auf diese Weise ist eine bessere Vergleichmäßigung des Geschwindigkeitsprofils im nachgeschalteten Feuerraum zu erreichen (vgl. Kap. 5).

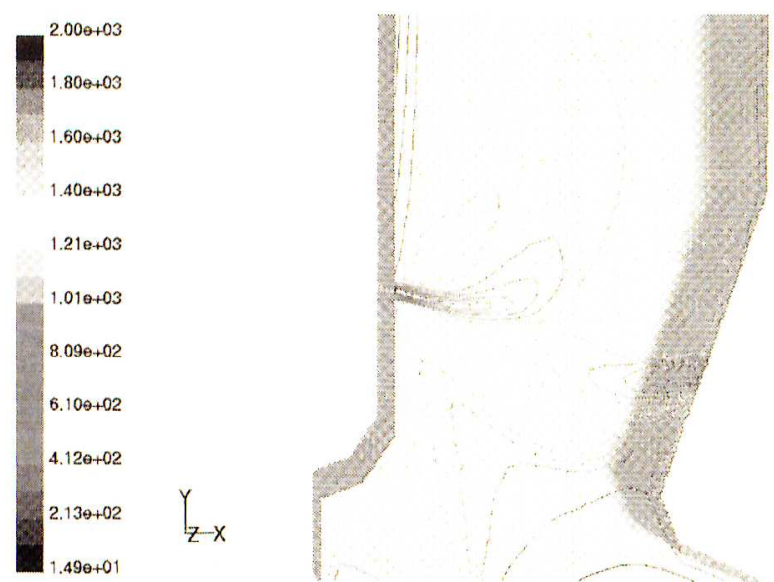

Abbildung 11: Konturen der Temperatur in ${ }^{\circ} \mathrm{C}$ (Symmetrie)

\section{Temperatur- / Wärmeübertragung}

Abbildung 11 zeigt die Temperaturverteilung im Bereich der Sekundärlufteindüsung und verdeutlicht, wie sich der Rauchgasstrom durch die Eindüsung der „kalten“ Sekundärluft (288K) abkühlt.

\section{Feuerraum}
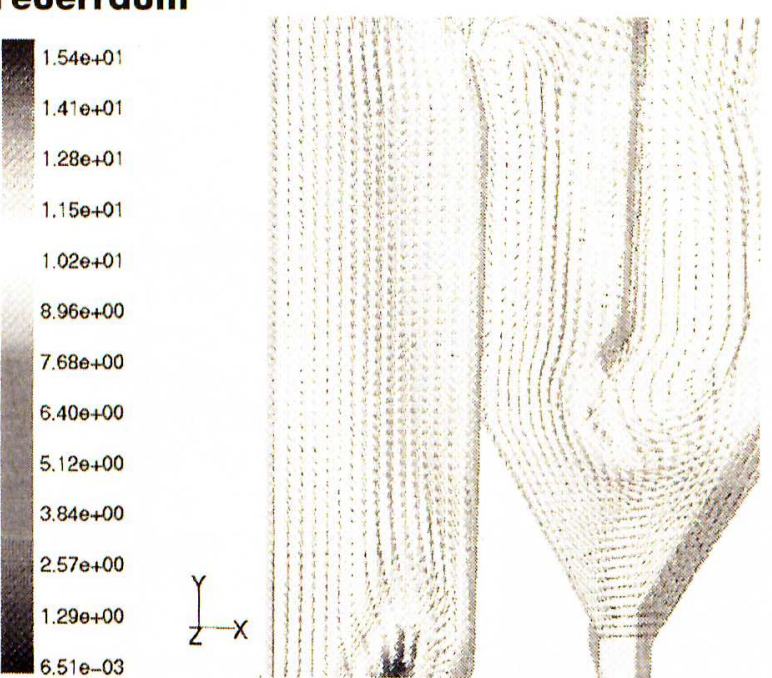

Abbildung 12: Geschwindigkeitsvektoren in m/s, Symmetrie (gefärbt durch den Betrag der Geschwindigkeit)

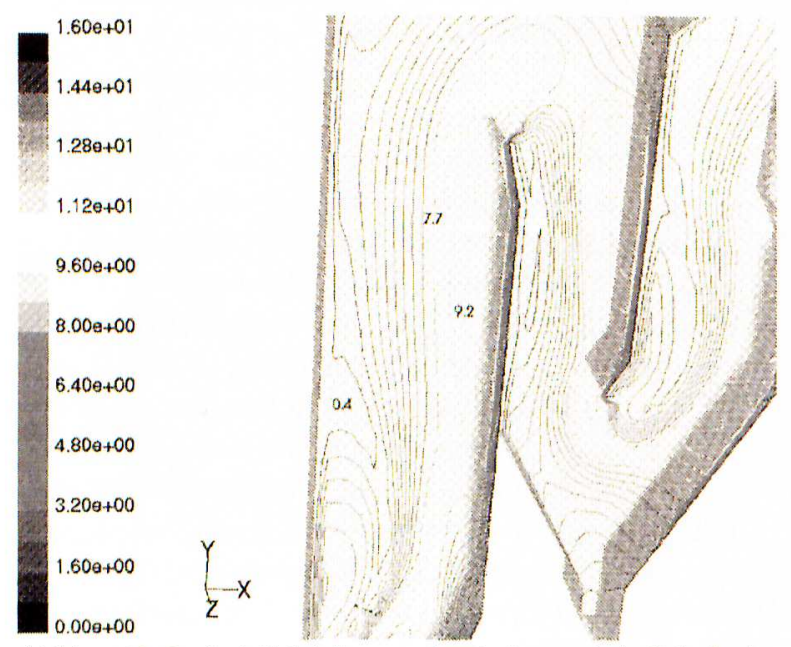

Abbildung 13: Geschwindigkeitskonturen in m/s, Symmetrie (gefärbt durch den Betrag der Geschwindigkeit)

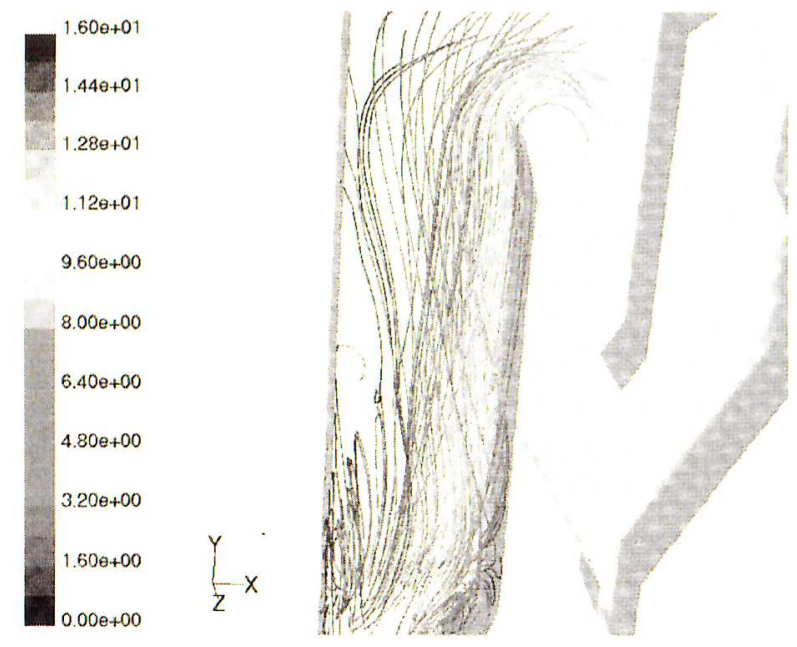

Abbildung 14: Stromlinien in m/s, Symmetrie (gefärbt durch den Betrag der Geschwindigkeit)

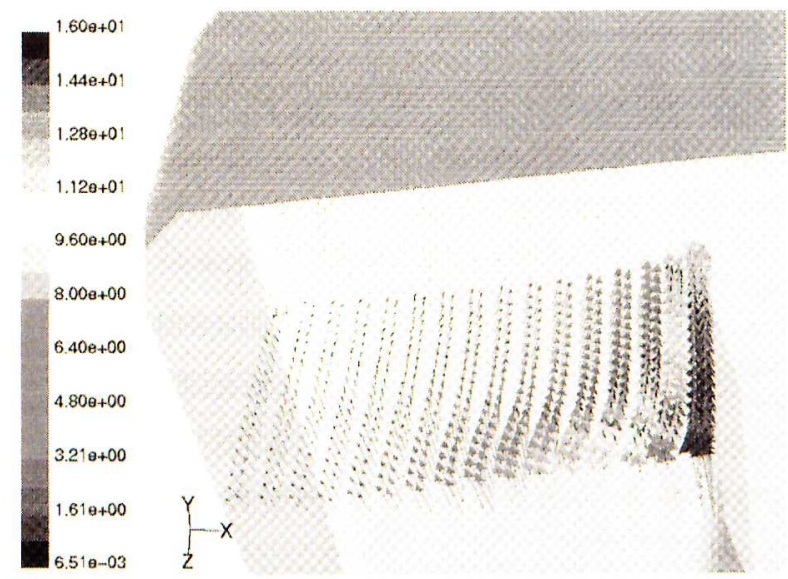

Abbildung 15: Geschwindigkeitsvektoren am Feuerraumaustritt in m/s (gefärbt durch den Betrag der Geschwindigkeit)

\section{Geschwindigkeitsfeld}

Die oben beschriebene Strömungseinschnürung führt dazu, daß sich eine Hauptströmung mit hoher Geschwindigkeit im Feuerraum ausbildet und an der Feuerraumrückwand anlegt. An der Feuerraumvorderwand bildet sich eine große Rezirkulationszone aus. Dieser Umstand führt dazu, daß das Feuerraumvolumen nur eingeschränkt für die Nachverbrennung genutzt wird, worauf später näher eingegangen wird.

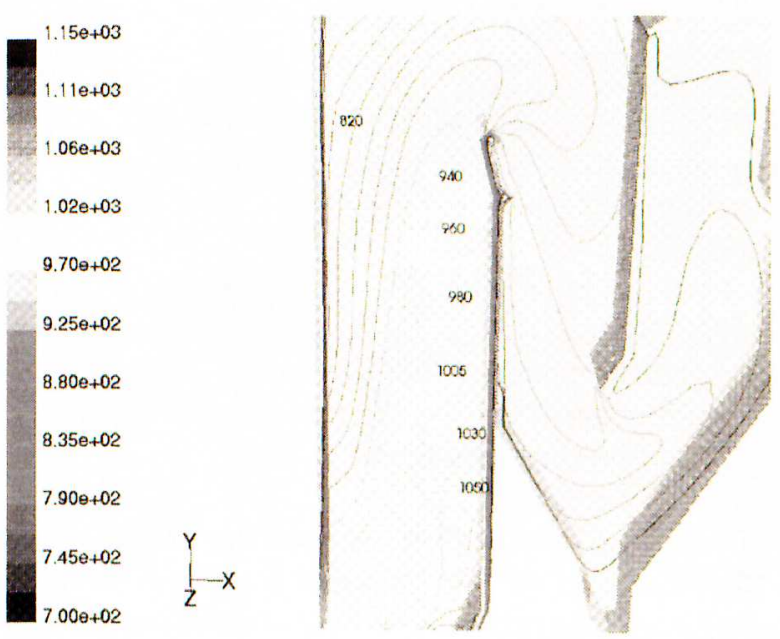

Abbildung 16: Konturen der Temperatur in ${ }^{\circ} \mathrm{C}$ (Symmetrie) 


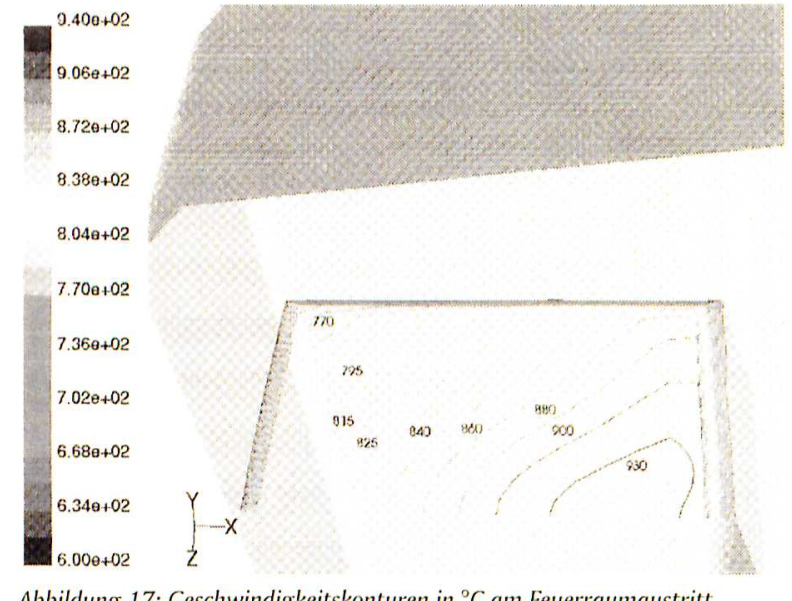

Abbildung 17: Geschwindigkeitskonturen in ${ }^{\circ} \mathrm{C}$ am Feuerraumaustritt

\section{Temperatur- / Wärmeübertragung}

Grundsätzlich korrespondiert das Geschwindigkeitsfeld bei erzwungenen konvektiven Strömungen mit dem Temperaturfeld. Bei der Temperaturverteilung im Feuerraum wird deutlich, daß sich kein gleichmäßiges Temperaturprofil im Feuerraum ausbildet. Die Hauptströmung an der Feuerraumrückwand weist wesentlich höhere Temperaturen als der Rezirkulationsbereich an der Feuerraumvorderwand auf.

\section{Staub / Erosion}

Im Bereich hoher Geschwindigkeiten werden große Staubpartikel mitgerissen. Bei den großen Partikeln ist die Wahrscheinlichkeit höher, daß diese noch nicht vollständig ausgebrannt sind und noch glühen. Treffen diese auf die Wände der Heizflächen, können sie lokal den Belägen Sauerstoff entziehen und somit die schuitzende Magnetitschicht abbauen und die Korrosion fördern.

Es ist anzunehmen, daß im Bereich der Hauptströmung, die Strömungsgeschwindigkeiten zwischen $7-9 \mathrm{~m} / \mathrm{s}$ aufweist, solche Partikel mitgerissen werden. Allerdings sind die stofflichen Umsatzraten in diesem Bereich aufgrund der hohen Temperaturen groß. Durch die geneigte Wand am Feurraumaustritt werden diese Partikel aufgrund der Zentrifugalkraftabscheidung mit hoher Wahrscheinlichkeit im Rezirkulationsbereich zurück in den Feuerraum getragen.

\section{Chemische Reaktion / Korrosion}

\section{reaktionstechnische Aspekte}

Unter dem Begriff Reaktionstechnik sind hier alle verfahrenstechnischen Vorgänge zusammengefaßt, die Nachstehendes betreffen:

erwünscht:

- Verbrennung (Oxidation)

unerwünscht:

- Schadstoffbildung (Kohlenmonoxid, Kohlenwasserstoffe, Stickoxide, Dioxine und Furane)

- Staubtransport (Katalyse)

- Korrosion

Wie eingangs beschrieben, wird nur das Rauchgas vom Entstehen über dem Feststoffbett bis zum Austritt aus dem Kessel betrachtet. Das Feststoffbett selbst wird hinsichtlich seiner reaktionstechnischen Eigenheiten nicht problematisiert. Trotzdem lassen sich die hier dargelegten Grundgedanken auch auf die Feststoff-Sauerstoff-Reaktionen übertragen.

Sowohl für die o.g. erwünschten wie unerwünschten chemischen Reaktionen gelten technische Prinzipien, die für ihr Zustandekommen und ihr nachhaltiges Ablaufen beachtet werden müssen:

- Den Edukten müssen hinreichend lange Reaktionszeiten (Verweilzeiten) zur Verfügung gestellt werden.

- Die Edukte muissen innig vermischt werden.

- Den erwünschten Reaktionen sind hohe, den unerwünschten niedrige Temperaturniveaus einzurichten. Druckeinfluisse können hier völlig ausgeschlossen werden.

Die genannten Prinzipien lassen sich in der Regel nicht getrennt voneinander einstellen.

In technischen System widersprechen sich die Forderungen an Verweilzeiten, Temperaturen und Mischbereiche häufig stark, so daß es gilt, optimale Bedingungen zu schaffen, d.h. hier die erwünschte Verbrennungsreaktion zu maximieren und den unerwünschten Reaktionseffekt zu minimieren.

Da unterschiedliche chemische Reaktionen auch i.A. sehr unterschiedliche Temperaturabhängigkeiten haben und unterschiedlichen kinetischen Mechanismen unterworfen sind, besteht die Möglichkeit, durch Temperaturstufung Erwünschtes und Unerwünschtes zu entkoppeln. Beispielsweise kann ein Verbrennungsvorgang zunächst auf niedrigem Temperaturniveau eingeleitet werden, indem unterstöchiometrische Bedingungen eingestellt werden. In weiteren Schritten wird so viel Sauerstoff nachgefuihrt, wie einerseits für die Verbrennung erforderlich ist und andererseits der gleichzeitig wirkenden Abkühlung der Flamme zur Einstellung gewisser max. Temperaturgrenzen entspricht. Letztere dienen dazu, die Bildung von thermischem $\mathrm{NO}$ zu vermeiden.

Wirbelgebilde, die nicht den gesamten Strömungsquerschnitt erfassen, sind reaktionstechnisch besonders problematisch. Die Rede ist von stehenden Wirbeln, wie sie in der Simulationsrechnung im Feuerraum über der Sekundärlufteindüsung, im 2. und 3. Zug und im Bereich des Vorverdampfers auftreten. Diese Wirbel vermischen zwar das Rauchgas, es wird aber kein wesentlicher Stoffaustausch mit der Hauptströmung herbeigefuihrt. Dies hat folgende Konsequenzen:

- Die Hauptströmung wird verdrängt, wodurch die mittlere Verweilzeit herabgesetzt wird.

- Die Geschwindigkeit der Hauptströmung steigt an, wodurch der Druckverlust erhöht wird.

- Im Wirbel halten sich die Edukte relativ lange auf, was zu einer starken Produktbildung führt (Ausbrand) und insofern zu einer erwünschten Erscheinung. Zugleich wird aber der Hauptströmung die Zeit für eine ausreichende Produktbildung genommen. Dieser Mangel wird, wie sich zeigen läßt, nicht durch hohe Produktbildung im Wirbel ausgeglichen.

Da der gesamte Reaktionsbereich nicht isotherm bzw. adiabat ist, sondern eine Wärmeabgabe an die gekuihlten Wände erfolgt, stellt sich in den wandnahen Wir- 
beln eine vergleichsweise niedrige Temperatur ein, die wesentlich zur Minderung des Ausbrandes beiträgt. Dieser Effekt verstärkt somit die negative Wirkung in Randbereichen auftretender Wirbel. Der Temperatureinfluß auf die stofflichen Umsatzraten ist nicht linear, sondern er geht rechnerisch exponentiell ein.

\section{Reaktionstechnik}

Vielfach wird der Einfluß der Verbrennung auf die Korrosion in den Beruihrungsheizflächen unterschätzt. Eine schlechte Verbrennung, wie sie im Fall A eintritt, führt dazu, daß Rauchgase mit einem hohen CO-Gehalt in die Beriihrungszuige eintreten. Diese können dort Schäden an Rohren mit hohen Rohrwandtemperaturen verursachen. Dabei wird die schützende Eisenoxidschicht reduziert und die Rohrwand schutzlos den aggressiven Stoffen ausgeliefert. Die sog. reduzierende Atmosphäre kann global wirken oder durch CO-reiche Strähnen entstehen.

\section{Umlenkung 1}
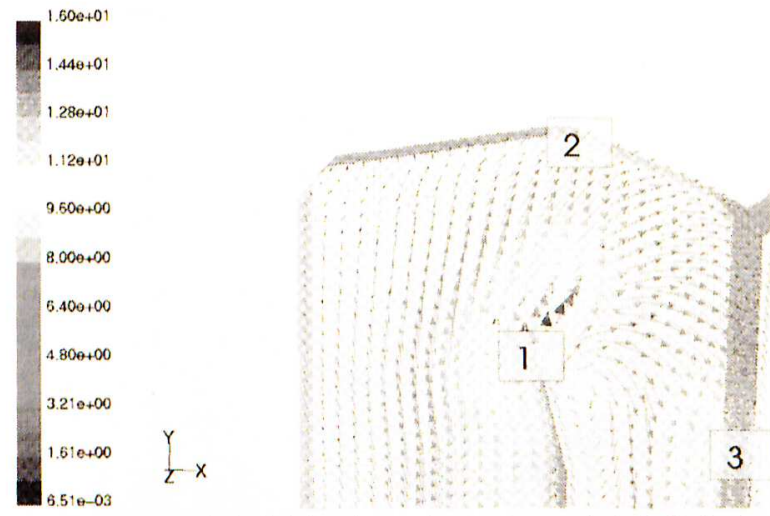

Abbildung 18: Geschwindigkeitsvektoren in m/s, Symmetrie (gefärbt durch den Betrag der Geschwindigkeit)

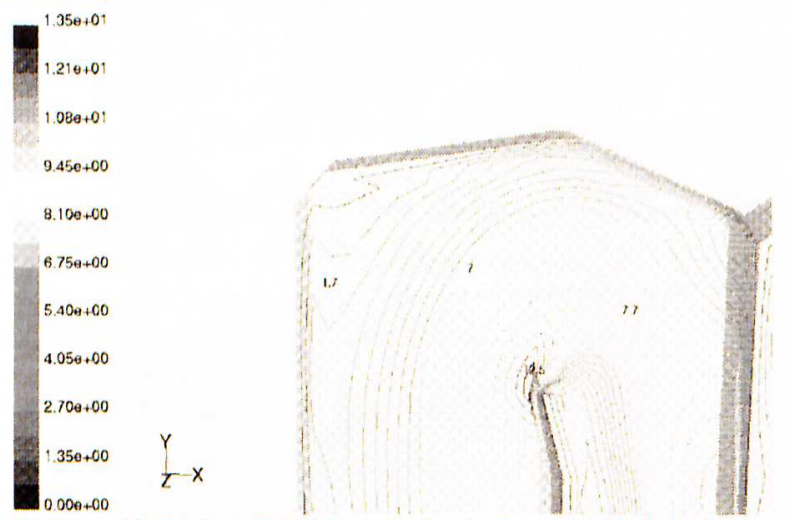

Abbildung 19: Geschwindigkeitskonturen in m/s, Symmetrie (gefärbt durch den Betrag der Geschwindigkeit)

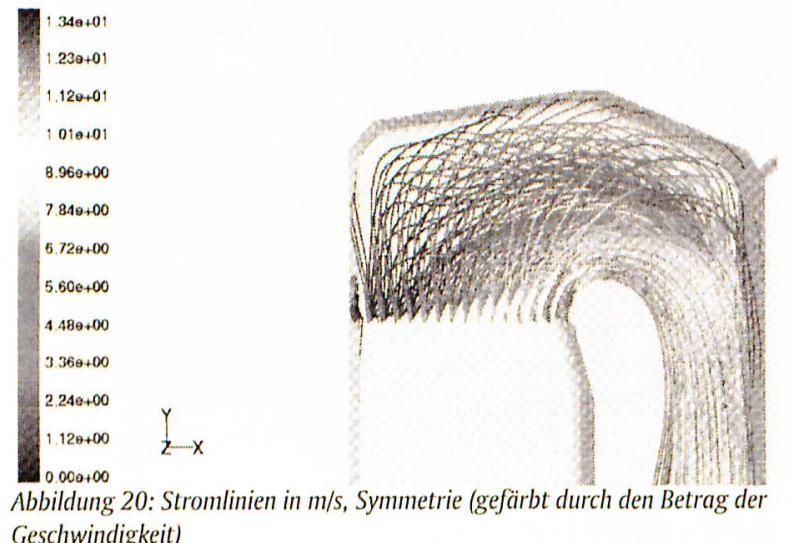

\section{Geschwindigkeitsfeld}

Erwartungsgemäß bildet sich hinter der geneigten Wand am Feuerraumaustritt ein Wirbel aus, der zu einer Rezirkulation des Rauchgases fuihrt. Die Wirbelausbildung läßt sich bei diesen scharfen Umlenkungen nicht verhindern. Diese wird in diesem Bereich allerdings kaum negative Folgen haben.

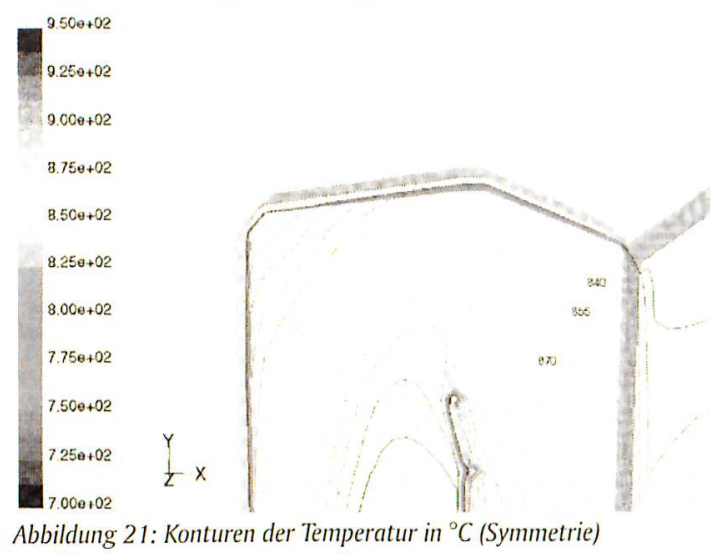

\section{Temperatur- / Wärmeübertragung}

Die scharfe Umlenkung fuihrt insgesamt zu einer Vergleichmäßigung der Temperatur.

\section{Staub / Erosion}

Abbildung 18 verdeutlicht, daß die mitgefuihrten Staubpartikel an den gekennzeichneten Stellen 1, 2 und 3 teilweise mit hohem Impuls auf die Wände auftreffen. Aus diesem Grund muß an diesen Stellen verstärkt mit Erosionskorrosion gerechnet werden.

\section{Umlenkung 2}

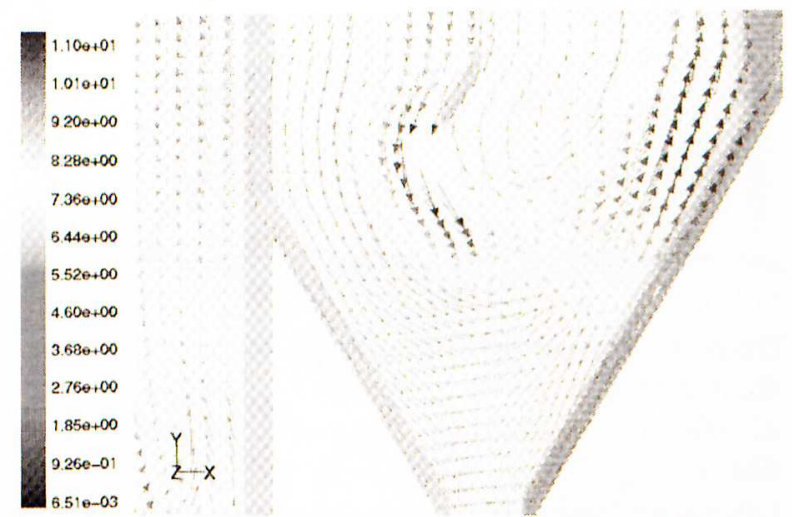

Abbildung 22: Geschwindigkeitsvektoren in m/s, Symmetrie (gefärbt durch den Betrag der Geschwindigkeit)

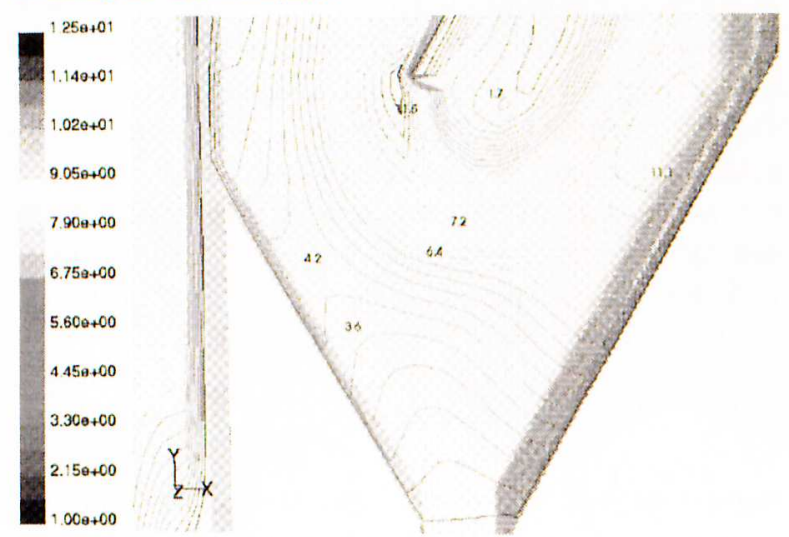

Abbildung 23: Konturen der Geschwindigkeit in m/s, Symmetrie (gefärbt durch den Betrag der Geschwindigkeit) 


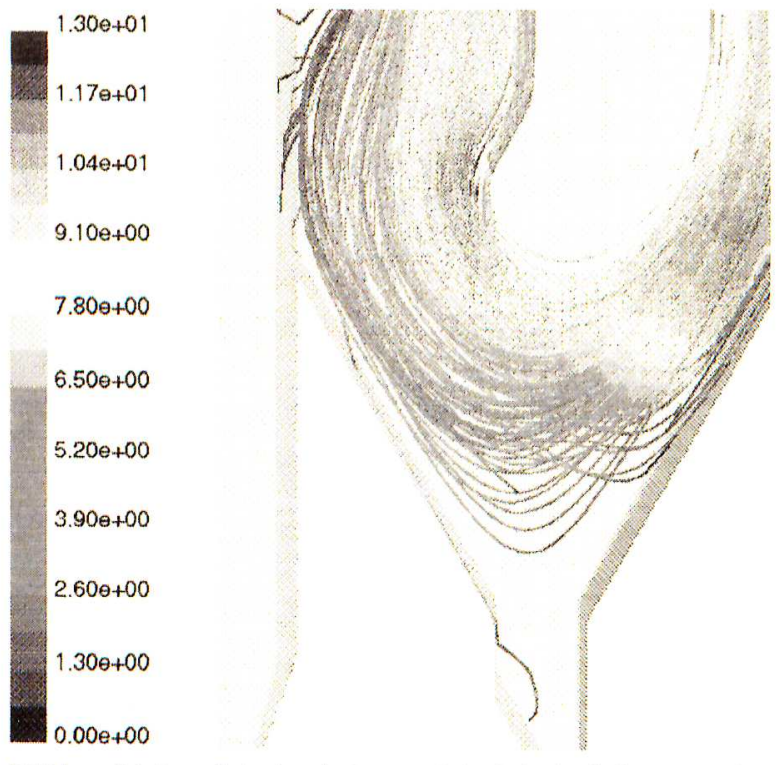

Abbildung 24: Stromlinien in m/s, Symmetrie (gefärbt durch den Betrag der Geschwindigkeit)

\section{Geschwindigkeitsfeld}

Das Geschwindigkeitsfeld im Übergangsbereich vom 2 in den 3 Zug muß prinzipiell unter dem Gesichtspunkt der gewïnschten Partikelabscheidung betrachtet werden.

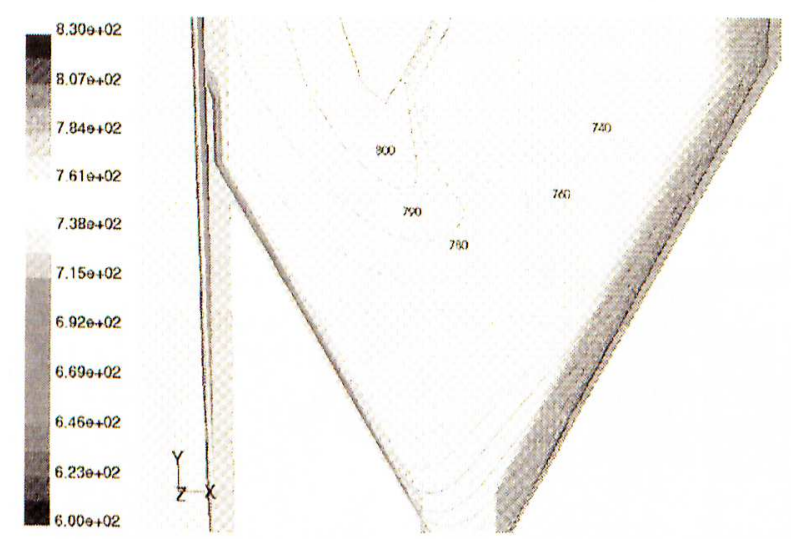

Abbildung 25: Konturen der Temperatur in ${ }^{\circ} \mathrm{C}$ (Symmetrie)

\section{Temperatur- / Wärmeübertragung Staub / Erosion}

Abbildung 22 verdeutlicht anschaulich die Wirksamkeit der geneigten Wand in Bezug auf die Partikelabscheidung. Die Rauchgasströmung wird durch die Querschnittsverringerung beschleunigt und bekommt einen Impuls in Richtung Feuerraumrïckwand. Die mitgefuihrten Partikel erfahren durch die Beschleunigung einen zusätzlichen Impuls und werden aufgrund ihrer Trägheit im Trichter abgeschieden. Die Strömungsgeschwindigkeit im unteren Teil des Trichters ist sehr niedrig, weshalb die Wahrscheinlichkeit gering ist, daß bereits abgeschiedene Partikel von der Strömung wieder aufgenommen werden.

\section{Eintrritt Berührungszüge}

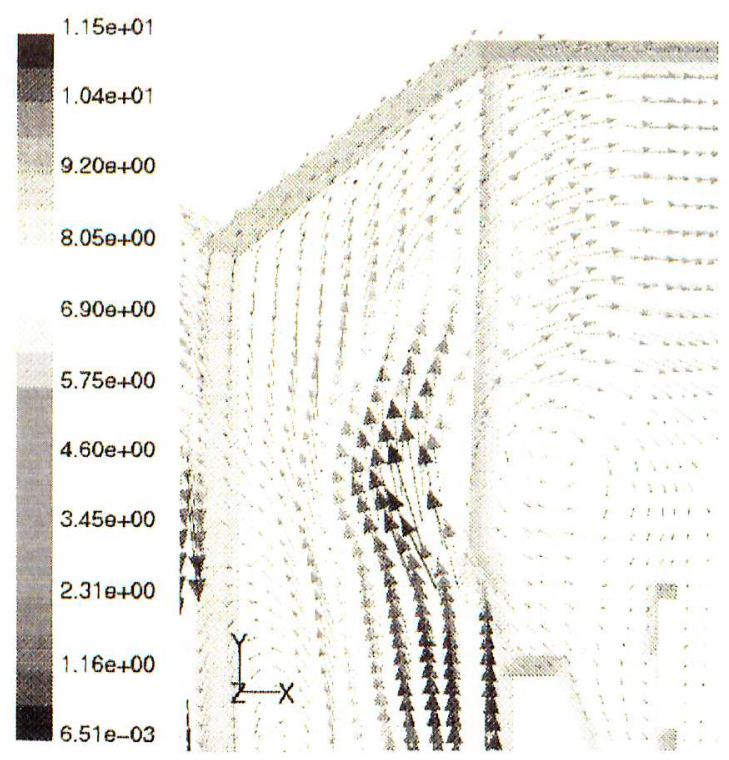

Abbildung 26: Geschwindigkeitsvektoren in m/s, Symmetrie (gefärbt durch den Betrag der Geschwindigkeit)

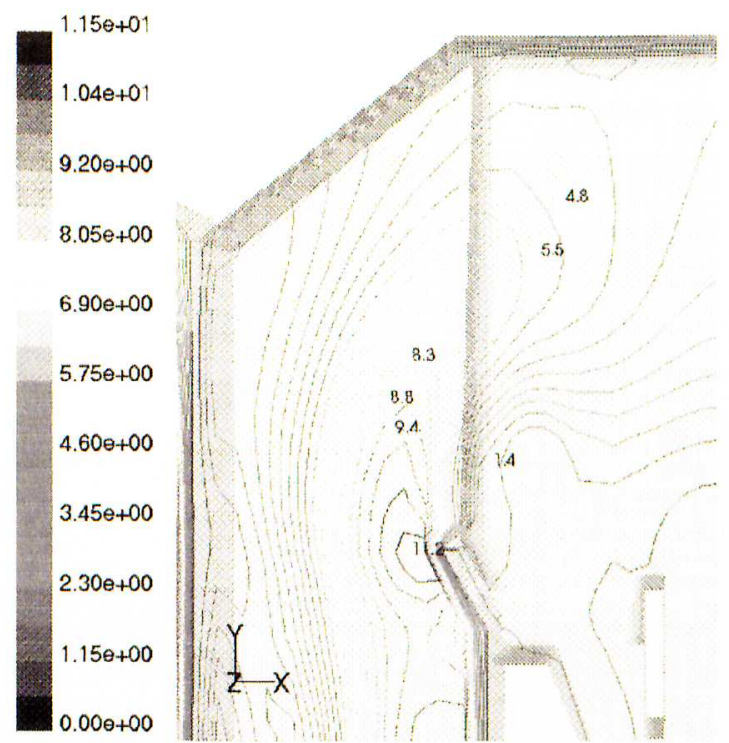

Abbildung 27: Geschwindigkeitskonturen in m/s, Symmetrie (gefärbt durch den Betrag der Geschwindigkeit)

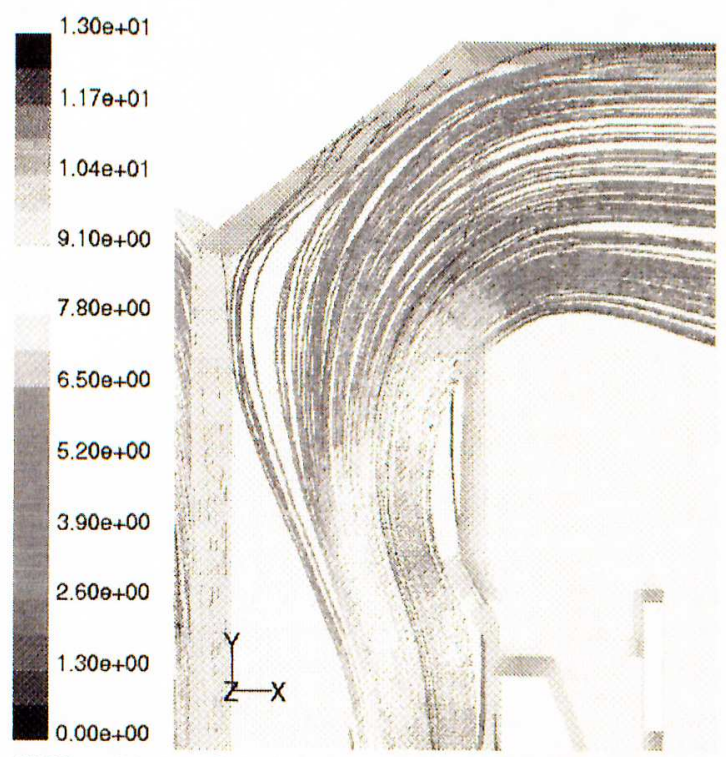

Abbildung 28: Stromlinien in m/s, Symmetrie (gefärbt durch den Betrag der Geschwindigkeit). 


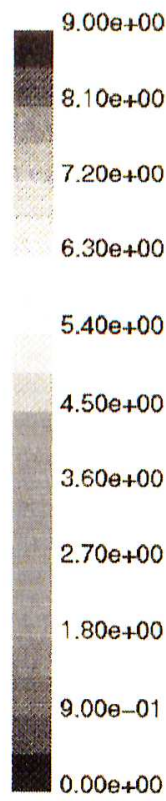

Abbildung 29: Geschwindigkeitsvektoren an Eingang des Übertragungsweges in m/s (gefärbt durch den Betrag der Geschwindigkeit)

\section{Geschwindigkeitsfeld}

Die Hauptströmung tritt mit hoher Geschwindigkeit im oberen Drittel des Eintrittsbereichs in die Beruihrungsziige ein.

In der unteren Hälfte des Strömungsquerschnitts bildet sich ein stehender Wirbel aus.

Durch die Verdampferheizfläche wird die Strömung allerdings vergleichmäßigt, wodurch die nachgeschalteten Heizflächen durch ein homogenisiertes Geschwindigkeitsfeld angeströmt werden.

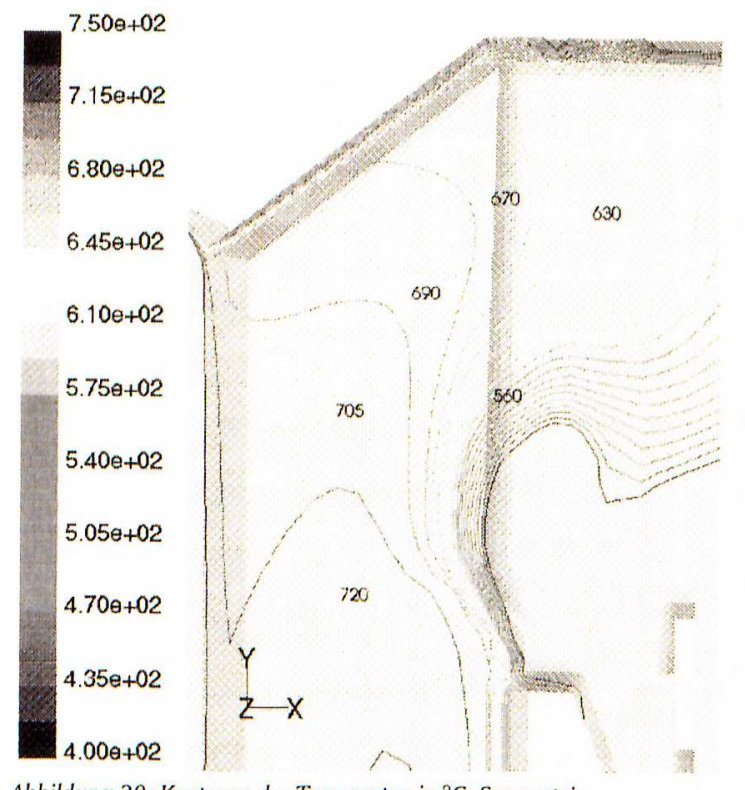

Abbildung 30: Konturen der Temperatur in ${ }^{\circ} \mathrm{C}$, Symmetrie

\section{Temperatur- / Wärmeübertragung}

Ausgehend von der Anströmung der Heizflächen kann von einer sehr ungleichen Wärmeübertragung in der ersten Verdampferheizfläche ausgegangen werden. Im Bereich der hohen Geschwindigkeit kommt es aufgrund der hohen Rauchgasgeschwindigkeit zu einer hohen Wärmeuibertragung, und die Rohrwandtemperatur steigt stark an. Im Gegensatz dazu ist die Wärmeüber-

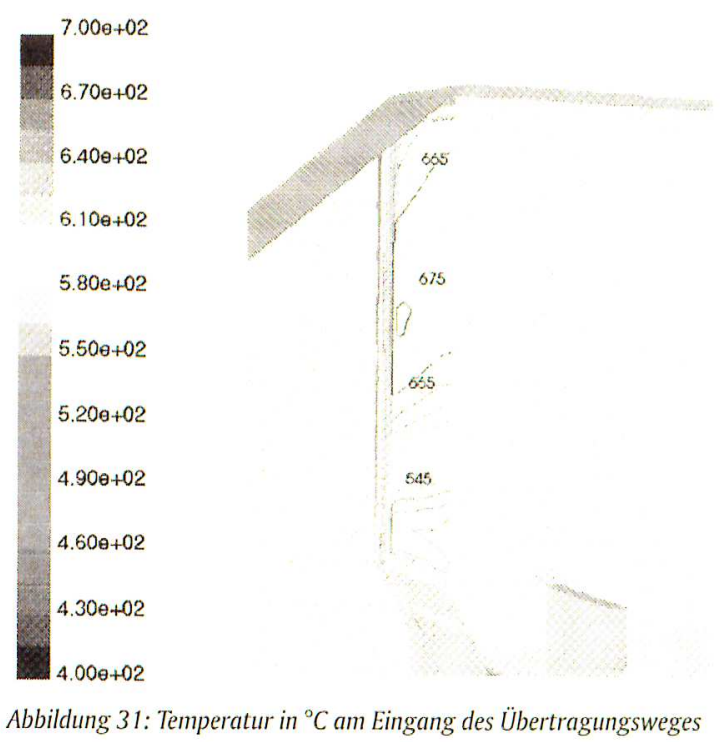

tragung im Bereich des stehenden Wirbels hinter der abgeknickten Wand gering.

Bilanziert man die innerhalb der Heizfläche übertragene Wärme, nimmt diese gegenuiber einer gleichmäßig angeströmten $a b$, da in den Bereichen geringer Geschwindigkeit deutlich weniger Wärme uibertragen wird (Wertigkeit der Heizfläche).

\section{Staub / Erosion}

Im Bereich hinter der abgeknickten Wand wird es sicherlich verstärkt zu Staubablagerungen kommen.

Erosionsschäden sind partiell im Bereich der Heizfläche zu erwarten, wo hohe Strömungsgeschwindigkeiten vorliegen und die Staubpartikel mit einem hohen Impuls auf die Rohrwand auftreffen. Allerdings ist die Wirksamkeit der Staubabscheidung am Trichter gut gelöst, weshalb die Staubfracht in der Strömung in diesem Bereich nicht mehr hoch sein wird.

\section{Modifikation der Sekundärluftzuteilung}
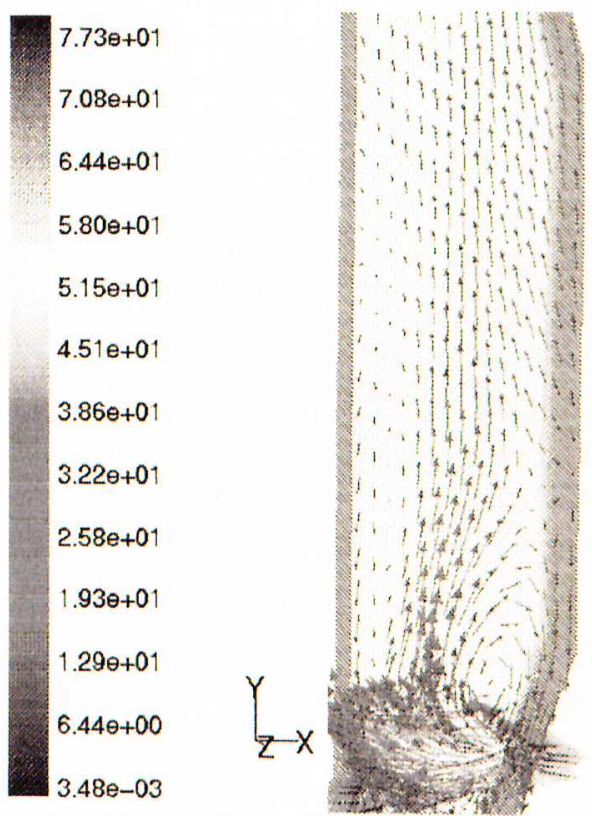

Abbildung 32a: Stromlinien gefärbt durch den Betrag der Geschwindigkeit in $\mathrm{m} / \mathrm{s}$ (Symmetrie) 
8.12e+01

$7.44 \theta+01$

$6.76 \theta+01$

$6.09 \mathrm{e}+01$

$5.41 e+01$

$4.73 e+01$

$4.06 e+01$

$3.38 \theta+01$

$2.71 \theta+01$

$2.03 \theta+01$

$1.35 e+01$

$6.76 \mathrm{e}+00$

$0.00 e+00$

Abbildung 32b: Geschwindigkeitsvektoren gefärbt durch den Betrag der Geschwindigkeit in m/s (Symmetrie)

Bei der Modifikation der Sekundärluftzuteilung wurde von einer einfachen Bilanzierung der durch die Sekundärluft eingebrachten Impulse in $x$-Richtung ausgegangen. Bringt man die Impulse von beiden Seiten ins Gleichgewicht, erhält man eine deutlich bessere Vergleichmäßigung der Geschwindigkeit im Feuerraum (Abbildung 32). Dadurch wird einerseits eine Vergleichmäßigung der Strömungsgeschwindigkeit erreicht, was zur Folge hat, daß die Schieflage der Temperatur (Abbildung 16) minimiert wird.

Insgesamt sind aus diesen Gründen bessere stoffliche Umsatzraten durch eine effizientere Nutzung des Feuerraumvolumens zu erwarten.

\section{Zusammenfassung}

Die vorgestellten Simulationsergebnisse, unterstreichen, daß durch CFD-Simulation die strömungs- und wärmetechnischen Vorgänge in Feuerräumen sehr real abgebildet werden können.

Das gewählte Design des Kessels wird aus strömungstechnischer Sicht die gestellten Anforderungen erfüllen.

Die abgknickte Wand im Bereich des Übergangs in die Berührungszuige könnte durch eine stärkere Abknickung oder durch ein weiteres Hineinragen der Wand in den Bereich des Strömungskerns optimiert werden, um den Wirbel, der sich an ihrer Rückwand im Bereich des Verdampfers ausbildet, zu verkleinern.

Eine Möglichkeit, den Sekundärluftbereich günstiger zu gestalten, ist ein Auseinanderziehen der Düsenreihen in vertikaler Ebene, um eine bessere Ausbildung lokaler Wirbel zu initiieren. Prinzipiell ist die Anordnung der Düsen sowie deren Anstellwinkel gut gewählt. Ein wichtiger Punkt in diesem Zusammenhang ist, daß immer eine Scherung zwischen den Strahlen gegenüberliegender Endüsungen erzeugt wird. Treffen die Strahlen frontal aufeinander, bildet sich in der vertikalen Ebene, ausgehend vom Kollisionpunkt der Strahlen, eine kalte Strähne aus.

Wie gezeigt kann die Aufteilung der Sekundärluftmengen durch eine Bilanzierung der eingebrachten Impulse günstiger gestaltet werden. Dies würde eine Vergleichmäßigung von Strömungsgeschwindigkeiten und Temperaturverteilungen nach sich ziehen, was mehrere positive Effekte initiieren würde:

- Verbesserung des Ausbrands,

- geringere Schadstoffbildung,

- geringere Staubfracht im Rauchgasstrom,

- höhere Verfügbarkeit der Anlage,

- geringerer Wartungsaufwand.

\section{Literatur}

[1] Görner, Klaus: technische Verbrennungssysteme, Springer Verlag; 1991.

[2] Linzer, W., Wiesenberger, J.: „Gestaltung von Rauchgaszügen in Müllverbrennungsanlagen“, VGB 72, H. 6 (1992), S. $527-530$

[3] Hellwig, U., Beyer, M., Nikolaus, H.: CFD (Computational Fluid Dynamics) Simulation des Berührungszuges eines Müllverbrennungskessels, Privatmitteilung der Fa. ERK Eckrohrkessel GmbH, Berlin.

\section{Autoren}

Prof. Dr.-Ing. Udo Hellwig

Technische Fachhochschule Wildau

Friedrich-Engels-Straße 63

15745 Wildau

Tel. (0 33 75) 508-170

Fax (0 33 75) 508-127

E-mail: hellwigu@vt.tfh-wildau.de

Dipl.-Ing. (FH) Hartwig Nikolaus

ERK Eckrohrkessel GmbH

Davoser Straße 2b

14199 Berlin

Tel. (0 30) 8977 46-17

Fax (0 30) 8977 46-46

E-mail: nnikolaus@eckrohrkessel.com 